\title{
Structural properties of the $\left[\mathrm{d}\left(\mathrm{G}_{3} \mathrm{~T}_{4} \mathrm{G}_{3}\right)\right]_{2}$ quadruplex: evidence for sequential syn - syn deoxyguanosines
}

\author{
Gary D.Strahan, Richard H.Shafer* and Max A.Keniry ${ }^{1}$ \\ Department of Pharmaceutical Chemistry, School of Pharmacy, University of California, \\ San Francisco, CA 94143, USA and ${ }^{1}$ Research School of Chemistry, The Australian National \\ University, GPO Box 4, Canberra, ACT 2601, Australia
}

Received July 5, 1994; Revised and Accepted October 25, 1994

\begin{abstract}
Two-dimensional 'H NMR studies on the dimeric hairpin quadruplex formed by $d\left(G_{3} T_{4} G_{3}\right)$ in the presence of either $\mathrm{NaCl}$ or $\mathrm{KCl}$ are presented. In the presence of either salt, the quadruplex structure is characterized by half the guanine nucleosides in the syn conformation about the glycosidic bond, the other half in the anti conformation, as reported for other similar sequences. However, ${ }^{1} \mathrm{H}$ NOESY and ${ }^{1} \mathrm{H}-{ }^{31} \mathrm{P}$ heteronuclear correlation experiments demonstrate that the deoxyguanosines do not strictly alternate between syn and anti along individual strands. Thus we find the following sequences with regard to glycosidic bond conformation: $5^{\prime}-\mathrm{G1}_{S} G 2_{S} G 3_{A} T 4_{A} T 5_{A^{-}}$

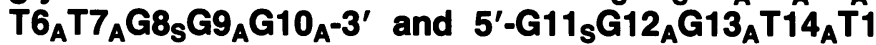
$5_{A} T_{16} \operatorname{Ti1}_{A} G 18_{S} G 19_{S} G 20_{A}-3^{\prime}$, where $S$ and $A$ denote syn and anti, respectively. This represents the first experimental evidence for a nucleic acid structure contairing two sequential nucleosides in the syn conformation. The stacking interactions of the resulting quadruplex quartets and their component bases have been evaluated using unrestrained molecular dynamics calculations and energy component analysis. These calculations suggest that the sequential syn-syn/ anti - anti and syn - anti quartet stacks are almost equal in energy, whereas the anti-syn stack, which is not present in our structure, is energetically less favorable by about $4 \mathrm{kcal} / \mathrm{mol}$. Possible reasons for this energy difference and its implications for the stability of quadruplex structures are discussed.
\end{abstract}

\section{INTRODUCTION}

Quadruplex nucleic acid structures, based on the guanine quartet, have been recognized for over three decades $(1,2)$. An intense research effort involving structural and physicochemical studies on model quadruplex systems $(3-15)$ has recently emerged not only because these structures provide an interesting example of DNA plasticity and polymorphism, but also due to their potential biological significance. For example, quadruplex structures have been implicated in telomere regions of chromosomes as well as in switch regions of immunoglobulin genes $(16,17)$. Additional recent evidence has been reported for a quadruplex-specific nuclease activity (18) as well as for a quadruplex-specific binding protein (19). Also, Sundquist and Heaphy (20) have described results consistent with a role for quadruplex formation in the association of the two copies of the RNA genome in the mature HIV virion. These observations provide suggestive evidence for the biological importance of quadruplex nucleic acid structures.

Quadruplex structures can be formed from either one, two, or four nucleic acid strands. There have been several high resolution NMR studies on four-stranded structures. For example, Cheong and Moore (21) have reported a structural analysis of [r(UGGGGU)] $]_{4}$, while Aboul-ela et al. (7) have described the DNA analog [d(TGGGGT) $]_{4}$. In both of these structures, the four strands are aligned in a parallel fashion, with all nucleosides in the anti conformation about the glycosidic bond. In the DNA quadruplex, the sugar conformation was mostly of the $S$ type, characteristic of B DNA, while in the RNA quadruplex, most of the nucleosides exhibited $\mathrm{N}$-type conformations, typical of RNA duplexes, although, interestingly, several were found in an $\mathrm{S}$ conformation.

Folded quadruplexes, mostly those composed of two strands, have also been described. Early NMR studies on $d\left(T_{2} G_{4}\right)_{4}$ showed evidence of slowly exchanging imino protons along with the presence of some guanine nucleosides in the syn conformation (22). More detailed studies on shorter sequences have been reported. Wang et al. (4) showed that, in the quadruplex formed by $d\left(G_{2} T_{5} G_{2}\right)$, syn-anti alternation occurred along sequential guanines on each strand. In addition, the deoxyguanosine at the $5^{\prime}$ end of the $\mathrm{T}$ loop was in the anti conformation, while that at the $3^{\prime}$ end was in the syn conformation. The same syn-anti alternation and deoxyguanosine conformations at the ends of the $\mathrm{T}$ loops have since been observed in quadruplexes formed by $\mathrm{d}\left(\mathrm{G}_{4} \mathrm{~T}_{4} \mathrm{G}_{4}\right)$, as studied by both NMR $(10-12)$ and $\mathrm{X}$-ray crystallography (8), and by d(GGTTTTCGG), also studied by NMR (5). Of particular interest is the different strand configurations in the dimeric hairpin quadruplex of $d\left(G_{4} T_{4} G_{4}\right)$ : in the $\mathrm{X}$-ray-derived structure (8), the planes formed by each of the two hairpin strands were parallel to each other, resulting in an edge-looped structure, while in the NMR-derived structure 
(10-12) they were perpendicular to each other, leading to a diagonally-looped structure (see Figure 1). Wang and Patel (14) have recently described an intramolecular quadruplex formed by $\mathrm{d}\left[\mathrm{AG}_{3}\left(\mathrm{~T}_{2} \mathrm{AG}_{3}\right)_{3}\right]$, based on the human telomere repeat sequence, containing three quartets in which there was also syn-anti alternation for deoxyguanosines along the entire strand. However, in this structure the deoxyguanosine at the $5^{\prime}$ end of the middle loop is in the syn conformation, while the one at the $3^{\prime}$ end is in the anti conformation. This is the opposite of what has been found in other folded structures, and may be an effect due to the presence of the adenosine at the $3^{\prime}$ end of the loop (see below).

In all of the NMR studies on either four-stranded or twostranded quadruplexes described above, there was a high degree of symmetry such that the strands making up the structure were magnetically equivalent. However, we have recently described the dimeric hairpin quadruplex, $\left[\mathrm{d}\left(\mathrm{G}_{3} \mathrm{~T}_{4} \mathrm{G}_{3}\right)\right]_{2}$, which exhibits an asymmetric structure with each strand possessing its own set of chemical shifts (23). While the quadruplexes formed by $\mathrm{d}\left(\mathrm{G}_{3} \mathrm{~T}_{4} \mathrm{G}_{3}\right)$ in either $\mathrm{NaCl}$ or $\mathrm{KCl}$ possess distinctly different one-dimensional ${ }^{1} \mathrm{H}$ spectra, in each case half of the guanine nucleosides are in the anti conformation about the glycosidic bond, while the other half are in the syn conformation, in agreement with results obtained for other folded quadruplexes. In this report, we now show that, in contrast to all previous studies on folded quadruplexes, the deoxyguanosines in the $\left[\mathrm{d}\left(\mathrm{G}_{3} \mathrm{~T}_{4} \mathrm{G}_{3}\right)\right]_{2}$ quadruplexes do not appear in strict syn-anti alternation along each strand. However, similar to other twostranded, folded quadruplexes, the deoxyguanosines at the $5^{\prime}$ and $3^{\prime}$ ends of the loop are observed to be in anti and syn conformations, respectively. This is the first nucleic acid structure characterized by two adjacent nucleosides in the syn conformation. We present unrestrained molecular dynamics calculations and energy component analysis for this novel conformational sequence and also discuss the overall structure of $\left[d\left(G_{3} T_{4} G_{3}\right)\right]_{2}$.

\section{MATERIALS AND METHODS}

\section{Experimental procedures}

The decamer $d\left(G_{3} T_{4} G_{3}\right)$ was synthesized and purified as described previously (23). The oligonucleotide was desalted either by dialysis alone or by chromatography on a Sephadex G25 column, followed by dialysis. The buffers used in the NMR studies were $10 \mathrm{mM} \mathrm{Na} 2 \mathrm{PO}_{4}, \mathrm{pH} 6.7$ for the sodium quadruplex and $10 \mathrm{mM}$ Tris, $\mathrm{pH} 7.0$, for the potassium quadruplex. Each quadruplex was formed by titrating with either $\mathrm{NaCl}$ or $\mathrm{KCl}$ while following changes in the imino/amino region of the NMR spectra at $20^{\circ} \mathrm{C}$ in a $90 \% \mathrm{H}_{2} \mathrm{O} / 10 \% \mathrm{D}_{2} \mathrm{O}$ solution. The quadruplex structures were considered completely formed at $30 \mathrm{mM} \mathrm{KCl}$ or $65 \mathrm{mM} \mathrm{NaCl}$. The concentration of DNA in both cases was $\sim 4 \mathrm{mM}$ (strand) in $400 \mu \mathrm{l}$.

Two-dimensional NMR spectra were recorded at $20^{\circ} \mathrm{C}$ on either a Varian VXR 500S or a GE GN500 spectrometer. Phasesensitive NOESY spectra in $100 \% \mathrm{D}_{2} \mathrm{O}$ were acquired in the pure absorption mode by the method of States et al. (24). Mixing times ranged from 50 to $240 \mathrm{~ms}$. Homonuclear correlation spectra were obtained using DQFCOSY and TOCSY pulse sequences. Spectra were apodized with a shifted sinebell weighting function. Each data set was zero filled to either a $2000 \times 1000$ or $4000 \times 2000$ real map and baselines were corrected and $t_{2}$ ridges removed by a two-dimensional version of a baseline correction

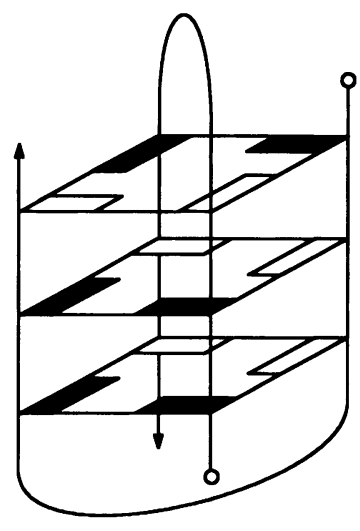

A

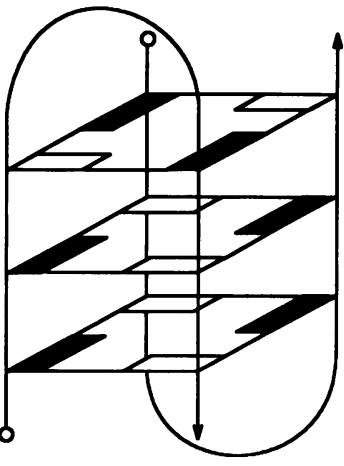

B
Figure 1. Schematic representation of possible hairpin dimer classes of structures: (A) diagonally-looped and (B) edge-looped. Shaded rectangles represent syn deoxyguanosines, clear rectangles represent anti deoxyguanines.

routine first proposed by Pearson (25). $\mathrm{A}^{1} \mathrm{H}-{ }^{31} \mathrm{P}$ heteronuclear COSY spectrum was acquired by the technique of Sklenar $e t$ al. (26) at $500 \mathrm{MHz}\left({ }^{1} \mathrm{H}\right)$ with spectral widths of $5 \mathrm{kHz}\left({ }^{1} \mathrm{H}\right)$ and $1.1 \mathrm{kHz}\left({ }^{31} \mathrm{P}\right) .2000$ real points were acquired in $t_{2}$ and 64 real points in $t_{1}$. The acquisition time was $3 \mathrm{~s}$ and 384 scans were acquired per increment.

\section{Theoretical procedures}

Stacking interaction energies of both the quartets and their component bases were determined by molecular mechanics and unrestrained molecular dynamics (MD) calculations in vacuo. All calculations were performed with the AMBER 4.0 suite of programs with previously reported force field parameters (27-29). Starting structures for these calculations, each consisting of a stack of three quartets, were generated by removing the thymine loops and one of the central quartets from the X-ray structure of $\left.\left[\mathrm{d}_{(} \mathrm{G}_{4} \mathrm{~T}_{4} \mathrm{G}_{4}\right)\right]_{2}$ (8) obtained from the Brookhaven Protein Data Bank. This produces a quadruplex similar to that found in the edge-looped structures, with three quartets, each having alternating syn, anti glycosidic conformations around the quartet $(\mathrm{S}-\mathrm{A}-\mathrm{S}-\mathrm{A}$, where $\mathrm{S}=$ syn and $\mathrm{A}=$ anti) but without strict syn and anti alternation sequentially along the strands. A second starting structure was then generated from the first one by switching the positions of two neighboring strands, thereby producing a quadruplex similar to that found in the diagonally-looped structure, where the glycosidic bond angles are $\mathrm{S}-\mathrm{S}-\mathrm{A}-\mathrm{A}$ around the quartet. For comparison, a third structure was generated by removing one of the terminal quartets from the original X-ray structure (8) to generate a quadruplex structure with strict sequential alternation of the glycosidic bond angles along the strand. As discussed below, this third structure was not observed in our experiments. Hexahydrated $\mathrm{Na}^{+}$counterions were added at a distance of 5 $\AA$ from the backbone phosphate groups to neutralize the negative charges. Anomalous model building artifacts were removed from each of these two structures by a combination of steepest descent and conjugate gradient energy minimization, followed by unrestrained MD where the temperature of the system was ramped up to $100 \mathrm{~K}$ over $20 \mathrm{ps}$. The final set of coordinates from 
these two runs was then used as input for the longer unrestrained MD runs.

In all calculations, the electrostatic energy was evaluated using the distance-dependent dielectric function, $\epsilon=R_{\mathrm{ij}}$, to mimic bulk solvent effects. These dynamics simulations were not run with explicit solvent, since only qualitative energy patterns were sought. Unrestrained molecular dynamics simulations were then performed for $150 \mathrm{ps}$ with a time step of $1 \mathrm{fs}$. All atoms within a $50 \AA$ radius were included in non-bonded interactions, and all bond lengths were constrained to their equilibrium values by the SHAKE algorithm (30). Translational and rotational motions were removed every $100 \mathrm{fs}$. Initial velocities were assigned to a Maxwellian distribution at $300 \mathrm{~K}$, and the system was kept at this temperature throughout the MD run by weak temperature coupling to a heat bath. No explicit restraints were used, including no hydrogen bonding restraints. Instead, the extent of hydrogen bonding was determined by the force field alone.

\section{RESULTS AND DISCUSSION}

\section{NMR experiments}

The non-exchangeable protons of the $\left[\mathrm{d}\left(\mathrm{G}_{3} \mathrm{~T}_{4} \mathrm{G}_{3}\right)\right]_{2}$ quadruplex were assigned with the assistance of NOESY, TOCSY and DQFCOSY spectra collected at $500 \mathrm{MHz}$. Although the 1D spectra of the $\mathrm{K}^{+}$and $\mathrm{Na}^{+}$forms of the $\left[\mathrm{d}\left(\mathrm{G}_{3} \mathrm{~T}_{4} \mathrm{G}_{3}\right)\right]_{2}$ quadruplex exhibit substantial differences (23), we observe that the sequential connectivity information is very similar for each form. Non-sequential or inter-strand ${ }^{1} \mathrm{H}-{ }^{1} \mathbf{H}$ contacts demonstrate greater variability between the two forms (data not shown). One characteristic common to both the $\mathrm{Na}^{+}$and $\mathrm{K}^{+}$ forms, illustrated in the base $-\mathrm{H} 1^{\prime}$ region of the NOESY spectra shown in Figure 2, is the presence of six guanine residues in the syn conformation (the $\mathrm{H} 8-\mathrm{H}^{\prime}$ intranucleoside distance of a syn guanine is $\sim 2.5 \AA$ while that for an anti guanine is $\sim 3.7$ $\AA$; the former produces noticeably more intense crosspeaks than the latter). Since syn deoxyguanosine residues in quadruplexes have only been observed in folded structures, the presence of these syn deoxyguanosines, along with equilibrium sedimentation and circular dichroism data from our earlier studies (23), provides compelling evidence that these quadruplexes are dimeric hairpin structures.

Several possible $5^{\prime}-3^{\prime}$ connectivities had to be considered before determining the sequential assignments for each strand. The case of sequential $5^{\prime}$ anti-3' anti deoxynucleosides, typically observed in most forms of regular B and A DNA, is characterized by $5^{\prime} \mathrm{H} 1^{\prime}, \mathrm{H} 2^{\prime}-3^{\prime} \mathrm{H} 8 / \mathrm{H} 6$ distance connectivities. The 5' syn-3' anti sequence, found in Z DNA and in hairpin-looped quadruplexes $(10-12)$, is characterized by $5^{\prime} \mathrm{H} 1^{\prime}, \mathrm{H} 2^{\prime}-3^{\prime}$ H8/H6 sequential distance connectivities, along with a weaker $5^{\prime} \mathrm{H} 8-3^{\prime} \mathrm{H} 1^{\prime}$ contact. The 5' anti-3' syn sequence is also seen in Z DNA and hairpin-looped structures, but exhibits weak interresidue NOEs. Finally, $5^{\prime}$ syn $-3^{\prime}$ syn pairs, which have not been seen before, are expected to have weak inter-residue NOEs, of which a 5' $\mathrm{H} 8-3^{\prime} \mathrm{H} 1^{\prime} \mathrm{NOE}$ might be observable at long mixing times.

We reported previously (23) that the imino proton spectrum of $\left[\mathrm{d}\left(\mathrm{G}_{3} \mathrm{~T}_{4} \mathrm{G}_{3}\right)\right]_{2}$ in $\mathrm{NaCl}$ or $\mathrm{KCl}$ exhibited 12 resonances in the frequency range characteristic of guanine quartet hydrogen bonding. This clearly indicates the presence of three fully formed guanine quartets and we have assumed this in making assignments. The initial steps of the sequential assignment
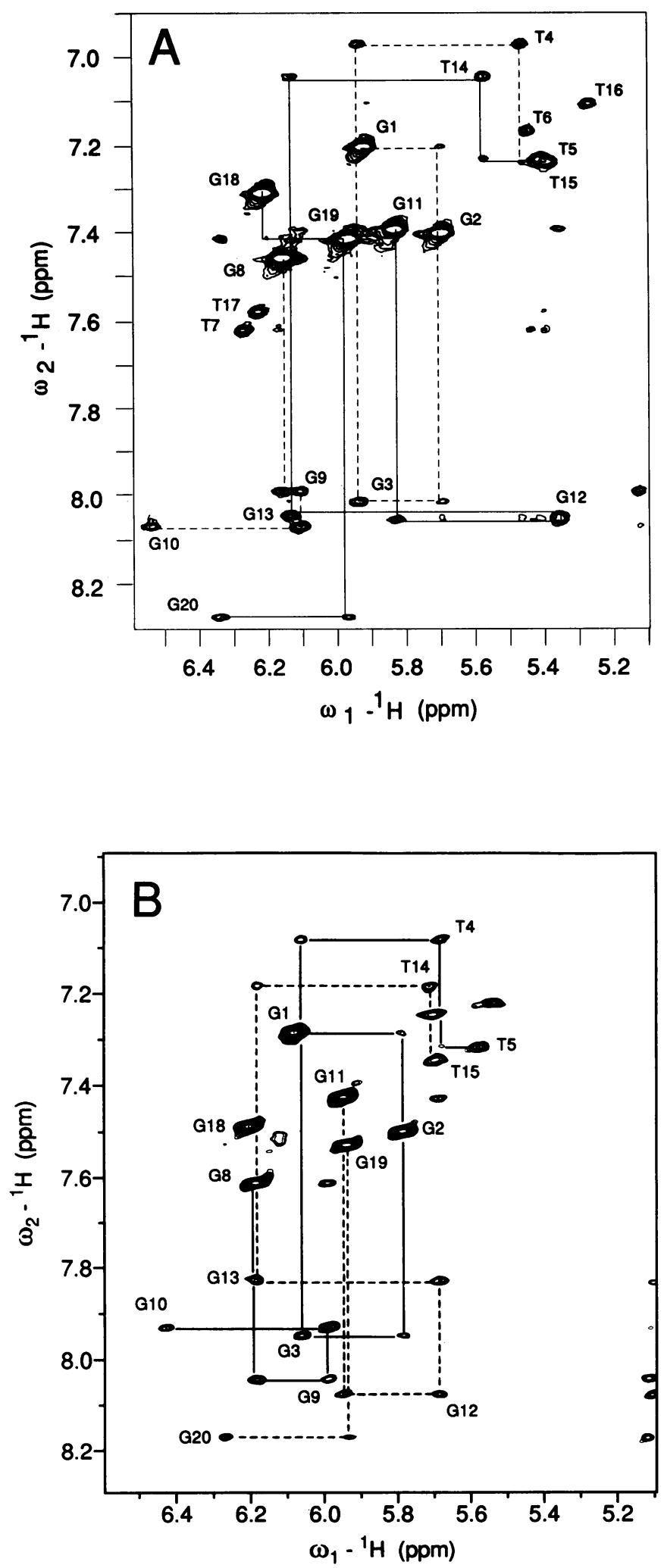

Figure 2. Expansion of the 2D NOESY spectrum illustrating the base $-\mathrm{H} 1^{\prime}$ crosspeaks of $\left[d\left(G_{3} T_{4} G_{3}\right)\right]_{2}$ at $20^{\circ} \mathrm{C}$ in $D_{2} O$. The sequential connectivity, where it can be followed, is traced for each strand with either a solid or a broken line. Data obtained in the presence of $\mathrm{NaCl}(\mathrm{A})$ at a $240 \mathrm{~ms}$ mixing time or $\mathrm{KCl}(\mathrm{B})$ at a $150 \mathrm{~ms}$ mixing time. 


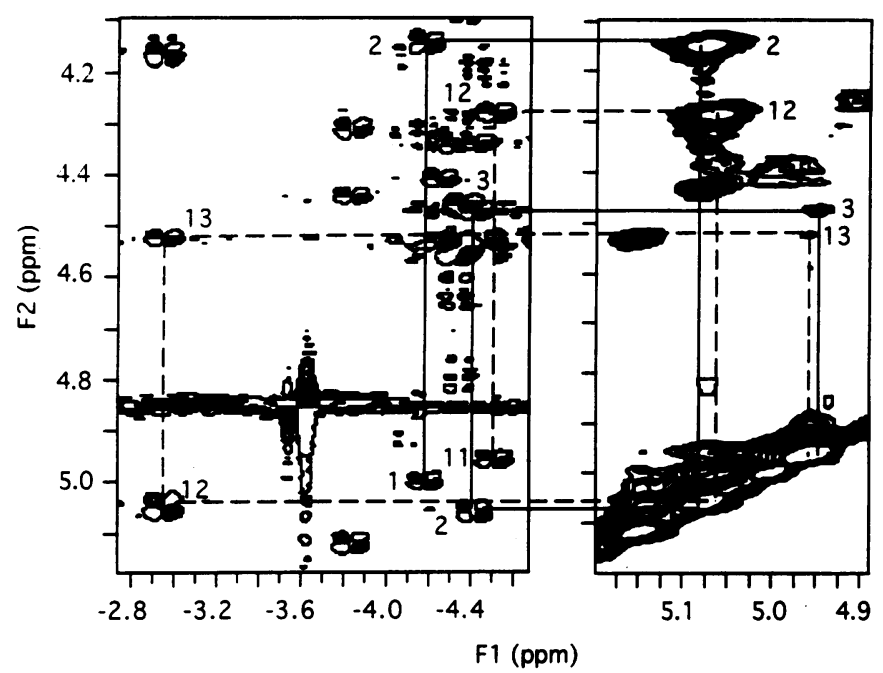

Figure 3. Combined ${ }^{1} \mathrm{H}-{ }^{31} \mathrm{P}$ heteronuclear correlation-TOCSY experiment measured for $\left[\mathrm{d}\left(\mathrm{G}_{3} \mathrm{~T}_{4} \mathrm{G}_{3}\right)\right]_{2}$ in $65 \mathrm{mM} \mathrm{NaCl}$ at $20^{\circ} \mathrm{C}$.

procedure, applied to spectra for both the $\mathrm{NaCl}$ and the $\mathrm{KCl}$ quadruplex forms, were accomplished with the base $-\mathrm{H} 1^{\prime}$ region of the NOESY spectra in $\mathrm{D}_{2} \mathrm{O}$ (Figure $2 \mathrm{~A}$ and $\mathrm{B}$ ) and confirmed by reference to the base $-\mathrm{H} 2^{\prime}, \mathrm{H} 2^{\prime \prime}$ region of the same spectra when sufficient spectral resolution was present. Two sequential $\mathrm{G}_{\mathrm{A}} \mathrm{T}_{\mathrm{A}} \mathrm{T}_{\mathrm{A}}$ strings (here subscripts $\mathrm{A}$ and $\mathrm{S}$ denote anti and syn, respectively) were initially identified in this region. Subsequently, one of these sequential $G_{A} T_{A} T_{A}$ strings could be extended in the 5 ' direction to $a G_{S} G_{A} G_{A} T_{A} T_{A}$ sequence, 'string one', while the other could be extended in the same direction to a $G_{S} G_{A} T_{A} T_{A}$ sequence, 'string two'. This established partial sequential connectivity within each strand, and left four syn and three anti guanines to assign. One of these syn guanines was at the $5^{\prime}$ end of the third $\mathrm{G}_{\mathrm{S}} \mathrm{G}_{\mathrm{A}} \mathrm{G}_{\mathrm{A}}$ string ('string three') and the other was at the 5 ' end of the last $G_{S} G_{A}$ string ('string four'). Strings three and four must lie on the $3^{\prime}$ end of the TTTT loop and identify two additional syn and all of the remaining anti guanines. In order to maintain Hoogsteen bonding in the central quartet, the anti guanines at the $3^{\prime}$ end of these last two strings must be the $3^{\prime}$ terminal guanines of each strand. This is supported by the observation that the sequential connectivity terminates at these two guanines, and leaves only two possible positions for the two remaining syn guanines, one on the $5^{\prime}$ side of string two and the other on the $5^{\prime}$ end of string four.

This set of connectivities implies that each of the guanines at the $3^{\prime}$ end of both TTTT loops is in the syn conformation. This is consistent with the absence of $5^{\prime}-T_{A}-G$ contacts of strong or medium intensity in this region since $5^{\prime}$-anti-syn-3' distances are not expected to be short. Also, there are two pairs of adjacent syn guanines, one of which possessed a weak $5^{\prime} \mathrm{H} 8-3^{\prime} \mathrm{H} 1^{\prime} \mathrm{NOE}$ contact (Figure $2 \mathrm{~A}$ and $\mathrm{B}$ ) which identified this guanine with string two, i.e. $5^{\prime}-G_{S} G_{S} G_{A} T_{A} T_{A}$. No sequential connectivity could be found for the other syn-syn pair in string four. The combining of the four strings to constitute complete oligonucleotide strands can be done in one of two ways, i.e. string one with string three and string two with string four or string one with string four and string two with string three. Only the second combination results in each strand containing three syn

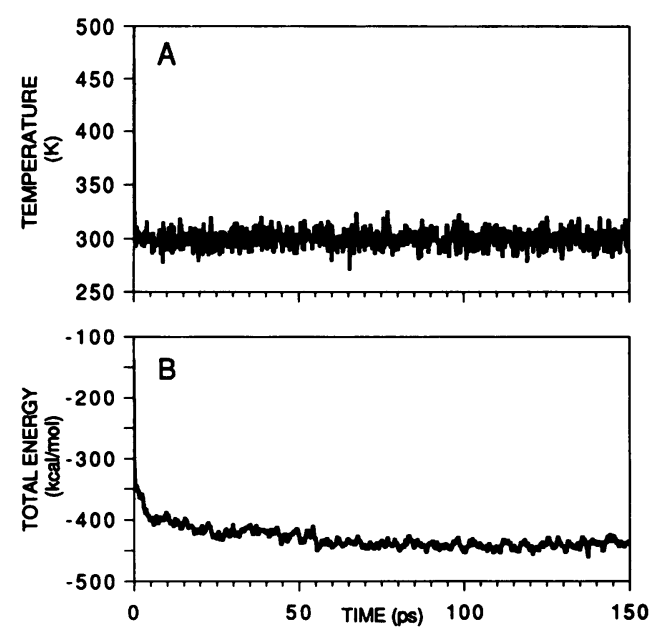

Figure 4. Time-dependent variations in (A) temperature and (B) total potential energy, for a representative 150 ps unrestrained MD run of a three quartet quadruplex in vacuo.

guanines. Furthermore, the first combination, but not the second, would lead to a quadruplex structure characterized by quartets containing both $\mathrm{S}-\mathrm{A}-\mathrm{S}-\mathrm{A}$ and $\mathrm{S}-\mathrm{S}-\mathrm{A}-\mathrm{A}$ configurations around the quartet. Such a structure is sterically unfeasible. This leads to the second combination and the following sequence assignment for the two strands in the dimeric hairpin quadruplexes: 5'-G1 ${ }_{S} \mathrm{G} 2_{S} \mathrm{G} 3_{\mathrm{A}} \mathrm{T} 4_{\mathrm{A}} \mathrm{T} 5_{\mathrm{A}} \mathrm{T} 6_{\mathrm{A}} \mathrm{T} 7_{\mathrm{A}} \mathrm{G} 8_{\mathrm{S}} \mathrm{G} 9_{\mathrm{A}} \mathrm{G10} \mathrm{A}^{-3} \mathbf{3}^{\prime}$ and $5^{\prime}-\mathrm{G} 11_{S} \mathrm{G} 12_{A} \mathrm{G} 13_{\mathrm{A}} \mathrm{T} 14_{\mathrm{A}} \mathrm{T} 15_{\mathrm{A}} \mathrm{T} 16_{\mathrm{A}} \mathrm{T} 17_{\mathrm{A}} \mathrm{G} 18_{\mathrm{S}} \mathrm{G} 19_{\mathrm{S}} \mathrm{G} 2 \mathrm{O}_{\mathrm{A}} \mathrm{-}^{\prime}$ '.

We have carried out a ${ }^{1} \mathrm{H}-{ }^{31} \mathrm{P}$ heteronuclear COSY experiment on $\left[\mathrm{d}\left(\mathrm{G}_{3} \mathrm{~T}_{4} \mathrm{G}_{3}\right)\right]_{2}$ in $\mathrm{NaCl}$ and these results, coupled with TOCSY data, have verified the connectivity in two regions of the stem. The $\mathrm{H}^{3}$ ' protons were identified by through-bond TOCSY connectivities to the $\mathrm{H} \mathrm{l}^{\prime}$ protons (data not shown). The H4' protons were subsequently identified by through-bond TOCSY connectivities to the $\mathrm{H}^{\prime}$ ' protons. Inter-residue $\mathrm{H}^{\prime}{ }^{\prime}-\mathrm{H}^{\prime}{ }^{\prime}$ correlations were made by utilizing the scalar coupling of the $\mathrm{H}^{\prime}$ ' proton on the $5^{\prime}$ side of the phosphorus atom and the $\mathrm{H}^{\prime}{ }^{\prime}$ atom on the $3^{\prime}$ side of the phosphorus atom. For reasons of clarity, we display only the important stem $\mathrm{G} 1_{\mathrm{S}} \mathrm{G} 2_{\mathrm{S}} \mathrm{G} 3_{\mathrm{A}}$ and $\mathrm{G} 11_{\mathrm{S}} \mathrm{G} 12_{\mathrm{A}} \mathrm{G} 13_{\mathrm{A}}$ correlations in a composite TOCSY $/{ }^{1} \mathrm{H}-{ }^{31} \mathrm{P}$ COSY spectrum shown in Figure 3. We can, in fact, sequentially assign $\mathrm{G} 1-\mathrm{T} 7, \mathrm{G} 9-\mathrm{G} 10$, and $\mathrm{G} 11-\mathrm{T} 17$. The segments $\mathrm{T} 7-\mathrm{G} 9$ and T17-G20 cannot be completed because of spectral overlap and weak or non-existent crosspeaks in the heteronuclear spectrum. However, there is a very weak contact in the homonuclear ${ }^{1} \mathrm{H}$ NOESY spectrum between $\mathrm{T} 7 \mathrm{H} 4{ }^{\prime}$ and $\mathrm{G} 8 \mathrm{H} 8$ protons, along with a similar contact between $\mathrm{T} 17 \mathrm{H} 4^{\prime}$ and G18H8, which confirms this overall strand connectivity.

Besides the direct evidence of sequential $5^{\prime}-\mathrm{G}_{\mathrm{S}} \mathrm{G}_{\mathrm{S}}{ }^{-3}$ ' stacking provided by the ${ }^{1} \mathrm{H}-{ }^{31} \mathrm{P}$ COSY experiment, we note that the observed intensities of the $\mathrm{G} 1_{\mathrm{S}} \mathrm{H} 1^{\prime}-\mathrm{G} 2_{\mathrm{S}} \mathrm{H} 8$ NOESY contacts are in agreement with an interproton distance of about $3.8 \AA$ (as determined by the relaxation matrix analysis discussed below). This is consistent with contacts between sequential bases, whereas cross-strand contacts would be too distant to be observed. For example, in the X-ray structure of $\left.\left[\mathrm{d}_{(} \mathrm{G}_{4} \mathrm{~T}_{4} \mathrm{G}_{4}\right)\right]_{2}$ (8) the comparable cross-strand distances are 7-8 $\AA$. In addition, the G9 ${ }_{\mathrm{a}} \mathrm{H} 1^{\prime}-\mathrm{G} 10_{\mathrm{A}} \mathrm{H} 8$, and the $\mathrm{G} 12_{\mathrm{A}} \mathrm{H} 1^{\prime}-\mathrm{G} 13_{\mathrm{A}} \mathrm{H} 8$ contacts follow 

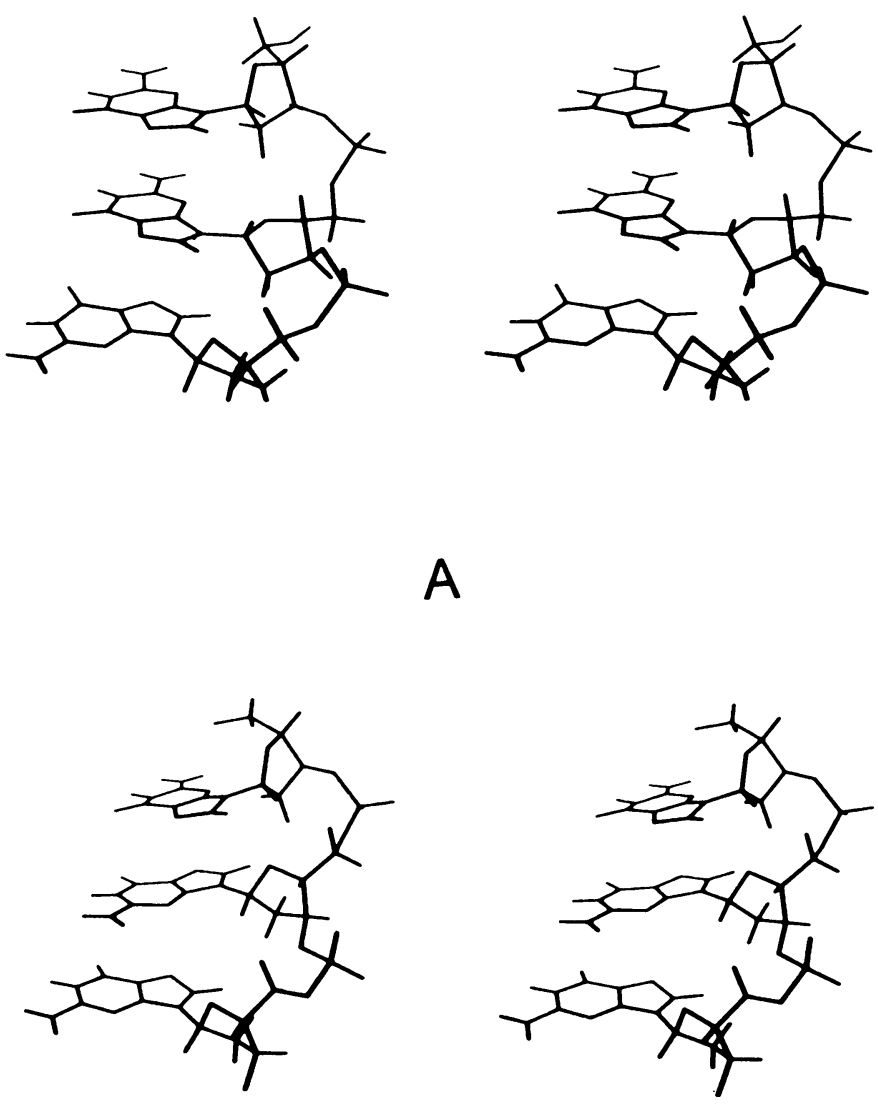

A

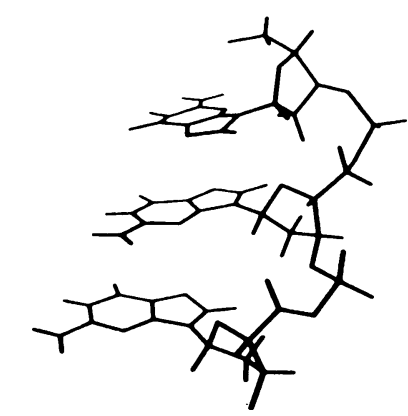

Figure 5. Stereo views of the trinucleotide segments $5^{\prime}-\mathrm{G}_{\mathrm{S}} \mathrm{G}_{\mathrm{S}} \mathrm{G}_{\mathrm{A}} 3^{\prime}$ (A) and $5^{\prime}-\mathrm{G}_{\mathrm{S}} \mathrm{G}_{\mathrm{A}} \mathrm{G}_{\mathrm{A}^{-}} 3^{\prime}$ (B) from the $\left[\mathrm{d}\left(\mathrm{G}_{3} \mathrm{~T}_{4} \mathrm{G}_{3}\right)\right]_{2}$ quadruplex. The $5^{\prime}$ end is at the top, the $3^{\prime}$ end is at the bottom.

all of the normal NOESY walk patterns which have been well established for stacked guanines in the anti conformation, as in B-DNA. Many of these connectivities are also observed in the $\mathrm{H} 8-\mathrm{H} 2^{\prime}, \mathrm{H} 2^{\prime}$, $\mathrm{H}^{\prime}$ ' regions of the spectra (data not shown). Finally, we do not observe any non-sequential GH8-GH8 NOE contacts, which is also consistent with the glycosidic bond sequence in our structure. When such contacts were detected in other quadruplex structures, they always occurred within an anti-syn (i.e. $5^{\prime}-\mathrm{G}_{\mathrm{A}} \mathrm{G}_{\mathrm{S}}-3^{\prime}$ ) quartet stack, which is absent in the $\left[\mathrm{d}\left(\mathrm{G}_{3} \mathrm{~T}_{4} \mathrm{G}_{3}\right)\right]_{2}$ quadruplex $(10,12,14)$. The corresponding distances in syn-anti (i.e. $5^{\prime}-\mathrm{G}_{\mathrm{S}} \mathrm{G}_{\mathrm{A}}-3^{\prime}$ ) quartet stacks are expected to be too long to detect $(8,14)$, as are those in the syn-syn/anti-anti (i.e. $5^{\prime}-\mathrm{G}_{\mathrm{S}} \mathrm{G}_{\mathrm{S}^{-}} 3^{\prime} / 5^{\prime}-\mathrm{G}_{\mathrm{A}} \mathrm{G}_{\mathrm{A}^{-}} 3^{\prime}$ ) stacks we have modeled. Thus, the evidence is overwhelming that this quadruplex species contains guanines with sequentially nonalternating glycosidic bond angles, resulting in two sets of $5^{\prime}-G_{S} G_{S^{-}}-3^{\prime}$ steps and two sets of $5^{\prime}-G_{A} G_{A}-3^{\prime}$ steps. This is a unique example of a DNA structure containing two adjacent syn nucleosides.

There are many possible dimeric hairpin quadruplex models consistent with the sequential assignment of syn and anti bases described above. In order to discriminate between these models, inter-strand and non-sequential intra-strand NOE contacts are required. Particularly useful are several NOE contacts (data not shown) observed in the NOESY spectrum of both the $\mathrm{Na}^{+}$and $\mathrm{K}^{+}$forms of the quadruplex between thymine methyl protons and the $\mathrm{H} 8, \mathrm{H}^{\prime}{ }^{\prime}$ and $\mathrm{H}^{\prime}$ ' protons of $\mathrm{G} 11$, and equivalent contacts in the other strand (similar types of loop-stem NOE contacts were also reported for the $\left[\mathrm{d}\left(\mathrm{G}_{4} \mathrm{~T}_{4} \mathrm{G}_{4}\right)\right]_{2}$ quadruplex (11). Such NOE contacts between the $5^{\prime}$ terminal guanines and thymine protons immediately exclude the edge-looped hairpin dimer structure with both loops at the same end of the quadruplex stem (23). This leaves two classes of quadruplex structures consistent with these data, illustrated in Figure 1, with variants arising from changes in strand polarity and hydrogen bonding orientation. Figure 1A is the three-quartet analog of the diagonally-looped structure for $\left[\mathrm{d}\left(\mathrm{G}_{4} \mathrm{~T}_{4} \mathrm{G}_{4}\right)\right]_{2}$ determined by NMR spectroscopy $(10-12)$, while Figure $1 \mathrm{~B}$ is the three-quartet analog of the edgelooped structure for $\left[\mathrm{d}\left(\mathrm{G}_{4} \mathrm{~T}_{4} \mathrm{G}_{4}\right)\right]_{2}$ determined by $\mathrm{X}$-ray crystallography (8). We note here that in our earlier report (23) we had indicated that $\left[\mathrm{d}\left(\mathrm{G}_{3} \mathrm{~T}_{4} \mathrm{G}_{3}\right)\right]_{2}$ could not take on diagonallylooped structures, as shown in Figure $1 \mathrm{~A}$, due to the observed asymmetry. This would only be true, however, for structures containing an even number of $G$ quartets and thus, for $\left[d\left(G_{3} T_{4} G_{3}\right)\right]_{2}$, which contains 3 quartets, such structures are indeed possible. Both classes of structures shown in Figure 1 are equally compatible with the non-alternating sequential glycosidic bond angles.

\section{Theoretical calculations}

Since the presence of these sequential syn guanines is highly unusual, we have investigated the stacking interaction energies of both the quartets and their component bases by analysis of results from unrestrained molecular dynamics calculations on the quadruplex stem in vacuo. There are two general classes of quadruplexes which differ in their arrangement of strands and in the order of guanosine glycosidic bond angles around the quartets. In the quartets derived from the diagonally-looped class, the order of deoxyguanosine conformations around each quartet is $\mathrm{S}-\mathrm{S}-\mathrm{A}-\mathrm{A}$ (where $\mathrm{S}=$ syn and $\mathrm{A}=$ anti), whereas in the edge-looped class, the order is $\mathrm{S}-\mathrm{A}-\mathrm{S}-\mathrm{A}$ (see Figure 1). The effect of sequential $5^{\prime}-G_{S} G_{S}-3^{\prime}$ steps within both types of quadruplex has been investigated. In addition, these energies are compared with the purely hypothetical three quartet stack with strict alternation of the glycosidic bond angles along the strand. The time-dependent energy variations of a typical $150 \mathrm{ps}$ unrestrained MD run are shown in Figure 4 and indicate that a minimum has been found. Coordinate sets were saved every 0.2 ps for each MD run, and the final 5 ps were averaged and energy minimized. The statistics of the energy component analysis were improved by repeating these MD runs a total of three times with different initial random seeds, thereby generating three slightly different structural variants for each of the quadruplex structures. Stereo views for representative $5^{\prime}-G_{S} G_{S} G_{A}-3^{\prime}$ and $5^{\prime}-\mathrm{G}_{\mathrm{S}} \mathrm{G}_{\mathrm{A}} \mathrm{G}_{\mathrm{A}}-3^{\prime}$ sequences are shown in Figure 5 .

The NOE intensities for the two sequentially non-alternating, unrestrained MD models were calculated and compared to the experimental intensities of a $150 \mathrm{~ms} 2 \mathrm{D}$ NOE spectrum of the quadruplex in $\mathrm{K}^{+}$by using the complete relaxation matrix algorithm CORMA $(31,32)$. An overall correlation time of 1.5 ns was utilized for these calculations. The $R$ factors listed in Table I reveal that all of the MD models are in fairly good agreement with most of the experimental intensities. The quality of these $R$ factors lends credence to the energies determined from these 
5452 Nucleic Acids Research, 1994, Vol. 22, No. 24

Table I. Comparison of unrestrained MD models to experimental data ${ }^{a}$

\begin{tabular}{lll}
\hline Quartet type in quadruplex & $R$ factors for 3 models & $R_{1 / 6}$ factors for 3 models $^{\mathrm{b}}$ \\
\hline $\mathrm{S}-\mathrm{S}-\mathrm{A}-\mathrm{A}$ & $0.377-0.383$ & $0.101-0.105$ \\
$\mathrm{~S}-\mathrm{A}-\mathrm{S}-\mathrm{A}$ & $0.344-0.371$ & $0.086-0.114$ \\
\hline
\end{tabular}

${ }^{a} R$ factors are calculated by comparison to 336 NOEs from both sides of the diagonal of 2D NOE spectra at $150 \mathrm{~ms}$ mixing time and only consider the $\mathrm{G}$ quartets.

${ }^{\mathrm{b}} R_{1 / 6}$ refers to the sixth root $R$ factor $(32,34)$

Table II. Average quartet stacking energies $(\mathrm{kcal} / \mathrm{mol})( \pm \text { standard deviation })^{\mathrm{a}}$

\begin{tabular}{llllll}
\hline $\begin{array}{l}\text { Quartet type within } \\
\text { quadruplex }\end{array}$ & $\begin{array}{l}\mathrm{G}-\mathrm{G} \text { step type(s) } \\
\text { between quartets }\end{array}$ & $E_{\mathrm{VDW}}$ & $E_{\text {elect }}$ & $E_{\mathrm{H}-\text { bond }}$ & $E_{\text {total }}$ \\
\hline $\begin{array}{l}\text { S-S-A-A sequentially } \\
\text { non-alternating }\end{array}$ & $\begin{array}{l}\text { syn-syn/anti-anti } \\
\text { syn-anti }\end{array}$ & $-47.4(1.2)$ & $0.63(0.21)$ & $-0.42(0.19)$ & $-47.2(1.5)$ \\
$\begin{array}{l}\text { S-A-S-A sequentially } \\
\text { non-alternating }\end{array}$ & $\begin{array}{l}\text { syn-syn/anti-anti } \\
\text { syn-anti }\end{array}$ & $-49.0(0.6)$ & $0.70(0.51)$ & $-0.25(0.04)$ & $-48.6(0.7)$ \\
$\begin{array}{l}\text { S-A-S-A sequentially } \\
\text { alternating }\end{array}$ & $\begin{array}{l}\text { anti-syn } \\
\text { syn-anti }\end{array}$ & $-48.9(0.4)$ & $0.44(0.49)$ & $-0.34(0.09)$ & $-48.3(1.1)$ \\
\end{tabular}

anteraction energies are based upon all those atoms within the aromatic bases of each quartet.

${ }^{a}$ The types of quartets (where $\mathrm{S}=$ syn and $\mathrm{A}=$ anti glycosidic bond angles) are related to the relative strand polarities $(8,12)$

Table III. Average base-base stacking energies $(\mathrm{kcal} / \mathrm{mol})( \pm \text { standard deviation })^{\mathbf{a}}$

\begin{tabular}{llllll}
\hline $\begin{array}{l}\text { Quartet type within } \\
\text { quadruplex }\end{array}$ & $\begin{array}{l}\text { G-G step type(s) } \\
\text { along strand }\end{array}$ & $E_{\mathrm{VDW}}$ & $E_{\text {elect }}$ & $E_{\mathrm{H}-\text { bond }}$ & $E_{\text {total }}$ \\
\hline $\begin{array}{l}\text { S-S-A-A sequentially } \\
\text { non-alternating }\end{array}$ & $\begin{array}{l}\text { syn-syn } \\
\text { anti-anti } \\
\text { syn-anti }\end{array}$ & $-9.11(0.66)$ & $+1.10(0.94)$ & $-0.028(0.008)$ & $-7.58(0.51)$ \\
& & $-7.58(0.85)$ & $+1.43(0.60)$ & $-0.025(0.011)$ & $-6.12(0.47)$ \\
S-A-S-A sequentially & syn-syn & $-7.64(0.47)$ & $-1.05(0.22)$ & $-0.013(0.007)$ & $-8.70(0.76)$ \\
non-alternating & $\begin{array}{l}\text { anti-anti } \\
\text { syn-anti }\end{array}$ & $-8.54(1.12)$ & $+1.31(0.85)$ & $-0.028(0.006)$ & $-7.26(0.47)$ \\
S-A-S-A sequentially & anti-syn & $-7.65(0.66)$ & $+0.91(0.49)$ & $-0.030(0.007)$ & $-6.84(0.66)$ \\
alternating & syn-anti & $-6.70(2.12)$ & $-1.09(0.28)$ & $-0.013(0.012)$ & $-8.70(1.16)$ \\
\hline
\end{tabular}

${ }^{a}$ Interaction energies are based upon all those atoms within the aromatic portion of each guanine base. The average is over each type of $G-G$ step within each of the three models per quadruplex type.

structures (see below). While it may be somewhat surprising that purely theoretical models can satisfy most of the experimental data, it must be kept in mind that these quadruplex structures exhibit a large amount of internal hydrogen bonding which is derived from the force field and results in an implicitly constrained structure. In addition, the initial structures for these MD simulations were models built from known experimental structures. We also note in Table I that both types of quadruplex quartet arrangements are nearly equal in their ability to predict NOE intensities. This certainly results from the fact that all of the NOE contacts within the quartet portion of the molecule are sequential in nature, and therefore reveal very little about the larger issue of strand arrangements.

After energy minimization of the MD averaged structures, energy component analysis was performed using the AMBER program ANAL on each of the six sequentially non-alternating structures (two classes, with three structural variants each) as well as the three strictly alternating structural variants. The stacking energies within each class were averaged together and their standard deviations determined (see Tables II and III). Since stacking interactions only involve the aromatic bases, these calculations only include those atoms found in the bases. In Table II we observe the effect of stacking quartets on top of each other.
The syn-syn/anti-anti stack refers to that orientation of quartets which has guanines with identical glycosidic bond angles on top of each other; thus, all of the sequential base steps are either $5^{\prime}-G_{s} G_{s^{-}} 3^{\prime}$ or $5^{\prime}-G_{A} G_{A^{-}} 3^{\prime}$. The syn-anti stack has one quartet reoriented with respect to the other one, such that all of the sequential steps are $5^{\prime}-G_{S} G_{A}-3^{\prime}$. For both classes of quadruplex quartets, the quartet stacking energies of the syn-syn/anti-anti quartet stack are approximately the same as those for the syn-anti quartet stack. Although the van der Waals interactions cause an estimated $1 \mathrm{kcal} / \mathrm{mol}$ preference for the syn-anti quartet stack, this difference is not significant, given the assumptions underlying these calculations and their computed standard deviations.

Quartet stacking includes both sequential and non-sequential base-base interactions; thus the stacking of two sequential bases represents a partial contribution to the quartet stacking. For these first two quadruplex structures with sequentially non-alternating glycosidic bond angles, the similar quartet stacking energies are reflected in the step-wise interactions of the individual bases. Table III reveals that the $5^{\prime}-G_{S} G_{A}-3^{\prime}$ base step has a slightly more favorable energy then the others, and this is followed by the $5^{\prime}-\mathrm{G}_{S} \mathrm{G}_{\mathrm{S}}-3^{\prime}$ base step, while the $5^{\prime}-\mathrm{G}_{\mathrm{A}} \mathrm{G}_{\mathrm{A}}-3^{\prime}$ step is higher in energy. The total energy spread between the highest and lowest energies of these steps is about $2.5 \mathrm{kcal} / \mathrm{mol}$. These differences 
in energy are the result of slight variations in the electrostatic and van der Waals terms (see Table III). Although these differences are statistically significant and tend to move up or down in concert, they probably do not reveal a significant difference in structural stability.

A visual comparison of the resulting non-alternating MD models can reveal probable structural reasons for these similar stacking energies. In both the $5^{\prime}-G_{S} G_{A}-3^{\prime}$ and $5^{\prime}-G_{S} G_{S}-3^{\prime}$ steps, the aromatic rings overlay each other in a slightly offset fashion, resulting in substantial $\pi-\pi$ stacking interactions. The guanines in the $5^{\prime}-G_{S} G_{A}-3^{\prime}$ stack have their dipoles oriented in nearly opposite directions, producing a geometry in which the fivemembered rings of the guanines in one of the quartets are stacked above the six membered rings of the other quartet. Since dissimilar atoms are above each other, the electrostatic interactions are improved, even while the van der Waals interactions are not as good as in the $5^{\prime}-\mathrm{G}_{\mathrm{S}} \mathrm{G}_{\mathrm{S}}-\mathbf{3}^{\prime}$ step. The guanines in the $5^{\prime}-\mathrm{G}_{\mathrm{S}} \mathrm{G}_{\mathrm{s}}{ }^{-3}{ }^{\prime}$ stack have nearly parallel dipole orientations with only a small horizontal displacement. Thus, although the van der Waals interactions seem favorable, there are more atoms of a similar type lying above each other, increasing the electrostatic component for this stack. The $5^{\prime}-G_{A} G_{A}-3^{\prime}$ step also has dipoles which are nearly parallel, but the guanine bases are significantly offset from each other compared to the other types of stacking, increasing its van der Waals interactions, and additionally, similar atom types overlay each other, resulting in a slightly less favorable total stacking energy. Force field calculations may be very sensitive to slight differences in molecular stacking geometries (28).

These energy calculations indicate that syn-syn/anti-anti quartet stacking is about equally favorable in energy compared to syn-anti stacking. This agrees with our experimental evidence, but does not explain why there are no $5^{\prime}-G_{a} G_{s^{-}} 3^{\prime}$ steps in this quadruplex; the only similar step is the $5^{\prime}-\mathrm{T}_{\mathrm{A}} \mathrm{G}_{\mathrm{S}^{-}} \mathbf{3}^{\prime}$ step which is found at the junction between the $3^{\prime}$-thymine of the loops and its adjacent guanine. We speculated that this type of stacking $\left(5^{\prime}-\mathrm{G}_{\mathrm{A}} \mathrm{G}_{\mathrm{S}^{-}} 3^{\prime}\right)$ is less stable than any of the other types, thereby explaining its absence in our experimental structures. To pursue this further, we performed similar calculations on a third quadruplex structure constructed from a three quartet stack with strict sequential alternation of the glycosidic bond angles. This structure includes both $5^{\prime}-\mathrm{G}_{\mathrm{S}} \mathrm{G}_{\mathrm{A}}-3^{\prime}$ and $5^{\prime}-\mathrm{G}_{\mathrm{A}} \mathrm{G}_{\mathrm{S}} \mathbf{3}^{\prime}$ base stacking within syn-anti and anti-syn quartet stacking, respectively. Visual inspection of the original $\mathrm{X}$-ray structure (8), from which this strictly alternating form was derived, suggests that the $5^{\prime}-\mathrm{G}_{\mathrm{A}} \mathrm{G}_{\mathrm{S}} \mathrm{-}^{\prime}$ ' stack is poorly aligned in terms of achieving stable $\pi-\pi$ stacking interactions, as the bases within this step appear to be almost completely displaced with respect to each other, and the backbone seems more stretched out to accommodate the changes in glycosidic bond angles.

The MD calculations on this third quadruplex structure indicated that it was significantly less stable than the other two structures when using the identical MD protocol. At $300 \mathrm{~K}$, the structure began to distort after about $90 \mathrm{ps}$. The distortion began as a disruption of the regular hydrogen bonding within the terminal quartet associated with the anti-syn quartet stack. The bases of this terminal quartet tilted inward and appeared to form transient hydrogen bonds and electrostatic interactions with the bases of the central quartet. By the end of $150 \mathrm{ps,} \mathrm{the} \mathrm{distortion}$ had sufficiently permeated the entire molecule as to render meaningless any attempt at a static energy analysis of the stacking. The other end of the molecule, involving the $5^{\prime}-G_{S} G_{A}-3^{\prime}$ quartet stack, remained intact, but was significantly distorted. Careful analysis of these MD runs revealed that this distortion appears to result from a fortuitous inter-quartet alignment of the guanine $\mathrm{H}$-bond donor and acceptor groups, as well as electrostatic interactions between the two quartets of the anti-syn quartet stack. This alignment seems to be unique to the anti-syn quartet step. Slight variations in the MD protocol were attempted, but no stable structure was possible at $300 \mathrm{~K}$ for the duration of the 150 ps MD simulation. As a result, we re-ran the MD experiments using only $200 \mathrm{~K}$ for $150 \mathrm{ps,} \mathrm{leading} \mathrm{to} \mathrm{much} \mathrm{more}$ stable structures for which energy component analysis could be performed. This problem did not arise with the non-sequentially alternating quadruplexes described earlier. It was also not a significant problem in any of the four quartet systems that we have examined (data not shown), even though these systems do include a single anti-syn central stack. The stability of the four quartet system may result from the very stable syn-anti stacks which cap both ends of the central anti-syn quartet stack. In addition, our calculations did not include coordinating central ions, which may improve the stability of the anti-syn stack. These results are also interesting in light of an experimental structure by Wang and Patel (14) which did have a three quartet quadruplex having strict sequential syn-anti alternation along the strand. This structure was determined at a temperature of $7^{\circ} \mathrm{C}$, which may have helped to stabilize an otherwise unstable anti-syn quartet stack.

Energy component analysis of the energy minimized, MD averaged structure at $200 \mathrm{~K}$ confirmed our speculation about the instability of the anti-syn quartet stack. We observe that the syn-anti quartet stack is $\sim 4 \mathrm{kcal} / \mathrm{mol}$ lower in energy than the anti-syn quartet stack. This is dominated by the van der Waals energy, where the two types of stacks differ by $\sim 6 \mathrm{kcal} / \mathrm{mol}$. This is expected considering the large extent to which the quartets are offset from each other. The sequential base-base interaction energies reveal additional insight concerning these stacks. As with the quartet stacking analysis, the van der Waals interactions favor the $5^{\prime}-\mathrm{G}_{\mathrm{S}} \mathrm{G}_{\mathrm{A}^{-}} \mathrm{3}^{\prime}$ base step by about $1.3 \mathrm{kcal} / \mathrm{mol}$. However, this gain is offset by a $1 \mathrm{kcal} / \mathrm{mol}$ higher electrostatic energy, resulting in very similar total base - base interaction energies for both the $5^{\prime}-\mathrm{G}_{\mathrm{A}} \mathrm{G}_{\mathrm{S}}-3^{\prime}$ and $5^{\prime}-\mathrm{G}_{\mathrm{S}} \mathrm{G}_{\mathrm{A}}-3^{\prime}$ steps. Using either the quartet - quartet or base - base analysis, the electrostatic energies favor the $5^{\prime}-G_{A} G_{S}-3^{\prime}$ stack over the $5^{\prime}-G_{S} G_{A}-3^{\prime}$ stack by a little more than $1 \mathrm{kcal} / \mathrm{mol}$. The anti-syn base stacking begins with the highest of the van der Waals stacking energies, but this difference is amplified when all quartet interactions are included. Clearly, the lack of favorable non-sequential interactions is very important in establishing the instability of the anti-syn quartet stack. The instability of this stack is also evidenced by the standard deviations in its energy components, which are consistently larger compared to those of the other steps. This is a reflection of the instabilities and distortions observed in the MD runs.

These calculations indicate that the syn-syn/anti-anti and syn-anti quartet stacks are essentially equally stable, and that the anti-syn stack is the least stable of all of them. The higher energy of the anti-syn stack is at least a partial explanation for its absence in this experimental system, and the MD experiments at $300 \mathrm{~K}$ suggest that when it is found in a three quartet quadruplex system it has the potential of being particularly destabilizing. Four quartet quadruplex systems, however, have capping syn-anti stacks, which may help stabilize their central anti-syn stack. 
Additional features of these folded quadruplexes which have not been evaluated in these studies because of their inherent complexity include the effects of internally coordinated cations and the thymine loops upon the arrangement and stability of the quartets. As noted earlier, almost all of the folded quadruplex structures analyzed experimentally to date have the guanine at the $5^{\prime}$ end of the loop in the anti conformation, while the one at the $3^{\prime}$ end is in the syn conformation (i.e. $5^{\prime}-G_{A}-$ loop$\left.\mathrm{G}_{\mathrm{S}^{-}} 3^{\prime}\right)$. This feature is conserved in the analysis of our quadruplexes as well. This suggests that $5^{\prime}-G_{A} T_{A^{-}} 3^{\prime}$ step may be energetically preferred over $5^{\prime}-\mathrm{G}_{\mathrm{S}} \mathrm{T}_{\mathrm{A}^{-}} \mathbf{3}^{\prime}$ step and/or that $5^{\prime}-T_{A} G_{S}-3^{\prime}$ step is preferred over $5^{\prime}-T_{A} G_{A}-3^{\prime}$ step. The only exception to this general pattern is found in the structure by Wang and Patel (14), which has the guanines before and after the central, diagonal loop in the opposite orientations (i.e. $5^{\prime}-G_{S}-$ loop $\left.-G_{A}-3^{\prime}\right)$; however, the two external loops of this structure follow the normal pattern. The Wang and Patel structure (14) is unique in that it possesses a 5'-terminal adenine extending beyond the quartet stem and each loop has an adenine at its $3^{\prime}$ end. The effects of cations, the guanine-loop junction and the loop configuration are clearly very important, but detailed analysis of their energetic contributions lies beyond the scope of this study.

\section{SUMMARY}

We have investigated the dimeric hairpin quadruplex formed by $d\left(G_{3} T_{4} G_{3}\right)$ and have found experimental evidence that it is unique in its sequential arrangement of deoxyguanosine conformations. We have determined that this sequential arrangement does not intrinsically imply or require either a diagonal- or edge-looped structural form, and our energetic calculations indicate that both structures should have very similar stacking energies. In our NOESY data obtained for the $\left[d\left(G_{3} T_{4} G_{3}\right)\right]_{2}$ quadruplex, there was no single crosspeak that could unambiguously distinguish the edge-looped from the diagonally-looped structures, in contrast to the case of the $\left[\mathrm{d}\left(\mathrm{G}_{4} \mathrm{~T}_{4} \mathrm{G}_{4}\right)\right]_{2}$ quadruplex (10). However, preliminary modeling of the NMR data of the sodium form clearly points to a diagonally-looped structure (Figure 1A); similar analysis of the NMR data of the potassium form is currently underway. Other workers have found that sodium and potassium can stabilize significantly different quadruplex structures in other oligonucleotide sequences $(16,33)$. Thus it may be possible for these two cations to stabilize different structural isomers of $\left[d\left(G_{3} T_{4} G_{3}\right)\right]_{2}$ as well. Extensive modeling and refinement of our NMR data will be reported elsewhere, but will not alter the energetic considerations discussed in this work.

Our energy analysis has shown that, for guanines, the syn-syn/anti-anti quartet stack is roughly equal in energy to the syn-anti quartet stack, while the anti-syn quartet stack is less favorable by $\sim 4 \mathrm{kcal} / \mathrm{mol}$. Although coordinated cations were not included in these calculations, the lower stability of the anti-syn stack is sufficient to account for its absence in our experimentally determined structure.

The results presented here add to a growing body of data for DNA quadruplexes and provide a unique example of adjacent syn deoxyguanosines not seen before. Strict sequential syn-anti alternation of the guanine conformation is not required in order to form hairpin-looped structures, but in each of the hairpin dimer structures solved to date, the purine preceding the first thymine of the loop is in the anti conformation. Thus, so far, the following primary sequence has always been observed: $5^{\prime}-\mathrm{G}_{\mathrm{S}}\left(\mathrm{G}_{\mathrm{X}}\right)_{n} \mathrm{G}_{\mathrm{A}} \mathrm{T}$ -
TTTG $_{\mathrm{S}}\left(\mathrm{G}_{\mathrm{X}}\right)_{n} \mathrm{G}_{\mathrm{A}}{ }^{-3^{\prime}}$ where $\mathrm{X}=\mathrm{S}$ or $\mathrm{A}$. However, the presence of a purine within the loop may complicate this issue (14). It is likely that the syn-syn sequence will occur in other dimeric hairpin quadruplexes composed of an odd number of guanine quartets. Since our calculations suggest that the syn-syn sequence is energetically similar to the anti-anti sequence for adjacent guanosines, it will be of interest to see if the $5^{\prime}-G_{s} G_{s}-3^{\prime}$ sequence arises in non-quadruplex structures as well.

\section{ACKNOWLEDGEMENTS}

We thank Dr C.Levenson for a generous gift of $d\left(G_{3} T_{4} G_{3}\right)$ used in some of the experiments described here, E.A.Owen for purification of additional oligonucleotide and assistance with the restrained molecular dynamics, and Wilson S.Ross for discussions regarding quadruplex modeling. This research was supported by NIH grant GM 51650, DHHS, and benefited from the use of NMR facilities at UCSF and at the Australian National University. We also acknowledge the Computer Graphics Laboratory, UCSF, supported by grant RR01081 from the Division of Research Resources, NIH, DHHS.

\section{Note added in proof}

An experimental study on the same structure has recently been reported by F.W.Smith, F.W.Lau and J.Feigon, Proc. Natl Acad. Sci. USA 91, 10546-10550 (1994).

\section{REFERENCES}

1. Gellert, M., Lipsett , M. N., and Davies, D. R. (1962) Proc. Natl. Acad. Sci. USA 48, 2013-2018.

2. Arnott, S. and Selsing, E. (1974) J. Mol. Biol. 108, 551-552.

3. Jin, R., Breslauer, K. J., Jones, R. A., and Gaffney, B. L. (1990) Science 250, 543-546.

4. Wang, Y., Jin, R., Gaffney, B. L., Jones, R. A., and Breslauer, K. J. (1991) Nucleic Acids Res. 19, 4619-4622.

5. Wang, Y., de los Santos, C., Gao, X., Greene, K., Live, D., and Patel, D. J. (1991) J. Mol. Biol. 222, 819-832.

6. Jin, R. Gaffney, B. L., Wang, C., Jones, R. A., and Breslauer, K. J. (1992) Proc. Natl. Acad. Sci. USA 89, 8832-8836.

7. Aboul-ela, F., Murchie, A. I. H., and Lilley, D. M. J. (1992) Nature, 360, $280-282$.

8. Kang, C., Zhang, X., Ratliff, R., Moyzis, R., and Rich, A. (1992) Nature 356, $126-131$.

9. Wang, Y. and Patel, D. J. (1992) Biochemistry 31, 8112-8119.

10. Smith, F. W. and Feigon, J. (1992) Nature 356, 164-168.

11. Smith, F. W. and Feigon, J. (1993) Biochemistry 32, 8682-8692.

12. Schultze, P., Smith, F. W., and Feigon, J. (1994) Structure 2, 221-233.

13. Wang, K. Y., McCurdy, S., Shea, R. G., Swaminathan, S., and Bolton, P. H. (1993) Biochemistry 32, 1899-1904.

14. Wang, Y. and Patel, D. J. (1993) Structure 1, 263-282.

15. Gupta, G., Garcia, A. E., Guo, Q., Lu, M., and Kallenbach, N. R. (1993) Biochemistry 32, 7098-7103.

16. Sen, D. and Gilbert, W. (1990) Nature 344, 410-414.

17. Blackburn, E. (1991) Nature 350, 569-572.

18. Liu, Z, Frantz, J. D., Gilbert, W., and Tye, B. K. (1993) Proc. Natl. Acad. Sci. USA 90, 3157-3761.

19. Weisman-Shomer, P. and Fry, M. (1993) J. Biol. Chem. 268, 3306-3312.

20. Sundquist, W. I. and Heaphy, S.(1993) Proc. Natl. Acad. Sci. USA 90, 3393-3397.

21. Cheong, C. and Moore, P. B. (1992) Biochemistry 31, 8406-8414.

22. Henderson, E., Hardin, C. C., Walk, S. K., Tinoco, I., Jr, and Blackburn, E. H. (1987) Cell 51, 899-908.

23. Scaria, P. V., Shire, S. J., and Shafer, R. H. (1992) Proc. Natl. Acad. Sci. USA 89, 10336-13340.

24. States, D. L., Haberkorn, R. A., and Ruben, D. J .(1982) J. Magn. Reson. 48, 286-292.

25. Pearson, G. A. (1977) J. Magn. Reson. 27, 265-272. 
26. Sklenar, V., Miyashiro, H., Zon, G., Miles, H. T., and Bax, A. (1986) FEBS Lett. 208, 94-98.

27. Pearlman, D. A., Case, D. A., Caldwell, J., Seibel, G. L., Singh, U. C., Weiner, P. K., and Kollman, P. A., (1991) AMBER 4.0 (UCSF), University of California, San Francisco, CA.

28. Weiner, S .J., Kollman, P. A., Nguyen, D. T., and Case, D. A. (1986) J. Comput. Chem. 7, 230.

29. Weiner, S. J., Kollman, P. A., Case, D. A., Singh, U. C., Ghio, C., Alagona, G., Profeta, S., Jr, and Weiner, P. K. (1984) J. Am. Chem. Soc. 106, $765-784$.

30. Ryckaert, J. P., Cicotti, G., and Berendsen, H. J. C. (1977) J. Comput. Phys. 23, 327-341.

31. Keepers, J. W. and James, T. L. (1984) J. Magn. Reson. 54, 404-426.

32. Borgias, B. A. and James, T. L. (1988) J. Magn. Reson. 79, 493-512.

33. Hardin, C. C., Henderson, E., Watson. T., and Prosser, J. K. (1991) Biochemistry 30, 4460-4472.

34. James, T. L. (1991) Curr. Opin. Struct. Biol. 1, 1042-1053. 